HD-THEP-99-29

\title{
Renormalization of lattice gauge theories with massless Ginsparg Wilson fermions
}

\author{
T. Reisz ${ }^{a, b *}$ and H. J. Rothe ${ }^{b}$, \\ ${ }^{a}$ SPHT-Service de Physique Theorique \\ CEA-SACLAY, \\ F-91191 Gif-sur Yvette Cedex, France \\ ${ }^{b}$ Institut für Theoretische Physik, \\ Universität Heidelberg, \\ Philosophenweg 16, \\ D-69120 Heidelberg, Germany
}

October 11, 2018

\begin{abstract}
Using functional techniques, we prove, to all orders of perturbation theory, that lattice vector gauge theories with Ginsparg Wilson fermions are renormalizable. For two or more massless fermions, they satisfy a flavour mixing axial vector Ward identity. It involves a lattice specific part that is quadratic in the vertex functional and classically irrelevant. We show that it stays irrelevant under renormalization. This means that in the continuum limit the (standard) chiral symmetry becomes restored. In particular, the flavour mixing current does not require renormalization.
\end{abstract}

\section{Introduction}

Recently, lattice regularization of chiral fermions have been proposed [1, 2] which circumvents the no-go theorem of Nielsen and Ninomiya [3]. In particular, if the Dirac operator satisfies the Ginsparg-Wilson relation [4] the fermion action posseses

${ }^{*}$ Supported by a Heisenberg Fellowship 
an exact chiral symmetry on the lattice [5]. It is free of fermion species doubling and local in a more general sense, at least for gauge fields that are sufficiently smooth on the scale of the UV cutoff [6]. In particular, $D$ decays exponentially fast, with a decay constant proportional to the lattice cutoff.

Renormalizability of lattice gauge theories was shown in [7]. Crucial ingredients are the BRS symmetry of the gauge fixed Faddeev-Popov action and of the functional measure. According to power counting on the lattice, the overall UV divergencies of the vertex functional are local lattice operators. They are removed by adding appropriate local counterterms to the lattice action. Linear gauge fixing that respects the discrete lattice symmetries implies that renormalization is achieved by renormalizing the fields and the gauge coupling constant. The renormalized theory becomes invariant under a (renormalized) BRS symmetry transformation. In the proof, Wilson fermions were chosen for the lattice realization of the Dirac operator. This avoids the species doubling problem and ensures that the lattice power counting theorem applies [8].

Another gauge invariant realization of the fermionic action that avoids the doubling problem are Ginsparg-Wilson fermions. The Faddeev-Popov trick applies along the usual lines. The resulting gauge fixed action becomes BRS invariant. Furthermore, locality in the more general sense mentioned above and the absense of species doubling allows one to apply the power counting theorem again. Hence the renormalizability proof of lattice gauge theories goes through also for the case of massive Ginsparg-Wilson fermions.

The renormalizability proof for massless fermions is not considerably more involved than for massive fermions because IR and UV singularities are properly disentangled. For two or more Ginsparg-Wilson fermions the theory possesses an exact chiral (flavour mixing) symmetry on the lattice. In this paper we show that this symmetry is preserved under renormalization. In particular, for two massless flavours, the renormalized vertex functional $\Gamma_{R}$ is found to satisfy the axial vector Ward identity

$$
\begin{gathered}
a^{4} \sum_{x}\left\{\frac{\partial \Gamma_{R}}{\partial a^{4} \psi(x)} \sigma_{\alpha} \gamma_{5} \psi(x)-\bar{\psi}(x) \sigma_{\alpha} \gamma_{5} \frac{\partial \Gamma_{R}}{\partial a^{4} \bar{\psi}(x)}\right\} \\
=a^{4} \sum_{x} \xi(a) \frac{\partial \Gamma_{R}}{\partial a^{4} \psi(x)} a \sigma_{\alpha} \gamma_{5} \frac{\partial \Gamma_{R}}{\partial a^{4} \bar{\psi}(x)}
\end{gathered}
$$

where $\left\{\sigma_{\alpha} \mid \alpha=1,2,3\right\}$ are the Pauli matrices acting on the flavour components of the fermion fields. $\xi(a)$ is such that the right hand side is UV finite to all orders and vanishes for $a \rightarrow 0$. With $\Gamma_{R}^{c}=\lim _{a \rightarrow 0} \Gamma_{R}$ we get

$$
\int d^{4} x\left\{\frac{\delta \Gamma_{R}^{c}}{\delta \psi(x)} \sigma_{\alpha} \gamma_{5} \psi(x)-\bar{\psi}(x) \sigma_{\alpha} \gamma_{5} \frac{\delta \Gamma_{R}^{c}}{\delta \bar{\psi}(x)}\right\}=0
$$

Hence, in the continuum limit one recovers the Ward identities associated with the continuum axial transformations. 
To avoid the technical complications of lattice QCD we will consider in the following the renormalization of an abelian lattice gauge theory, with two flavours of massless Ginsparg-Wilson fermions. This model preserves the main properties required for proving renormalizability also of non-abelian gauge theories with massless Ginsparg-Wilson fermions. The main inputs going into the proof are gauge or BRS invariance, the flavour mixing axial vector Ward identity associated with an exact chiral symmetry and the applicability of the power counting theorem.

\section{General framework}

Consider the partition function

$$
Z=\int \prod_{x \in a \mathbb{Z}^{4}}\left(d \psi(x) d \bar{\psi}(x) \prod_{\mu} d U(x ; \mu)\right) \exp \left(-S_{W}(U)-S_{f}(U, \psi, \bar{\psi})\right)
$$

on the hypercubic lattice $a \mathbb{Z}^{4}$, with $a$ the lattice spacing. $\psi$ is a 2 -flavour, Dirac spinor field,

$$
d \psi(x)=\prod_{f=1}^{2} \prod_{\alpha=1}^{4} d \psi_{f \alpha}(x)
$$

and $d U$ is the Haar measure on $U(1) . S_{W}(U)$ is the Wilson plaquette action

$$
S_{W}(U)=\frac{1}{2 g^{2}} \sum_{x \in a \mathbb{Z}^{4}} \sum_{\mu \neq \nu=0}^{3}\left(1-U(x ; \mu) U(x+a \widehat{\mu} ; \nu) U(x+a \widehat{\nu} ; \mu)^{-1} U(x ; \nu)^{-1}\right) .
$$

The fermion action is given by

$$
S_{f}=a^{4} \sum_{x \in a \mathbb{Z}^{4}} \bar{\psi}(x) D[U] \psi(x) .
$$

The lattice Dirac operator $D$ is translation invariant and is supposed to satisfies the Ginsparg Wilson relation

$$
\gamma_{5} D+D \gamma_{5}=a D \gamma_{5} D
$$

For $U(x ; \mu)=\exp i \operatorname{iag} A_{\mu}(x), D$ allows for a small $A$ expansion

$$
\begin{gathered}
D[\exp i a g A](x, y)=\sum_{n \geq 0} \frac{1}{n !} a^{4 n} \sum_{z_{1}, \ldots, z_{n}} \sum_{\mu_{1}, \ldots, \mu_{n}} D_{\mu_{1} \ldots \mu_{n}}^{(n)}\left(x, y \mid z_{1}, \ldots, z_{n}\right) \\
\cdot A_{\mu_{1}}\left(z_{1}\right) \cdots A_{\mu_{n}}\left(z_{n}\right) .
\end{gathered}
$$

The Fourier transforms $\widetilde{D}^{(n)}$ of the coefficient functions $D^{(n)}$ are analytic functions about zero momentum. 
Finally, for $a \rightarrow 0$, the action becomes

$$
\begin{aligned}
& S_{W}(U)+S_{f}(U, \psi, \bar{\psi}) \simeq \int d^{4} x\left\{\frac{1}{4} \sum_{\mu, \nu=0}^{3}\left(\partial_{\mu} A_{\nu}(x)-\partial_{\nu} A_{\mu}(x)\right)^{2}\right. \\
& \left.+\bar{\psi}(x) \sum_{\mu=0}^{3} \gamma_{\mu}\left(\partial_{\mu}+i g A_{\mu}\right) \psi(x)\right\}
\end{aligned}
$$

A possible solution of (7) that satisfies these conditions is given by [2, 5]

$$
\begin{aligned}
D(U) & =\frac{1}{a}\left(1-\mathcal{D}\left(\mathcal{D}^{+} \mathcal{D}\right)^{-1 / 2}\right) \\
\mathcal{D} & =1-a D_{W}
\end{aligned}
$$

where $D_{W}$ is the Wilson Dirac operator,

$$
\begin{aligned}
& D_{W}=\frac{1}{2 a} \sum_{\mu=0}^{3}\left[\left(\gamma_{\mu}-1\right) D_{\mu}^{c}+\left(\gamma_{\mu}+1\right) D_{\mu}^{c *}\right] \\
& \left(D_{\mu}^{c} \psi\right)(x)=U(x ; \mu) \psi(x+a \widehat{\mu})-\psi(x), \\
& \left(D_{\mu}^{c *} \psi\right)(x)=\psi(x)-U(x-a \widehat{\mu})^{-1} \psi(x-a \widehat{\mu}),
\end{aligned}
$$

and $\widehat{\mu}$ the unit vector in the positive $\mu$ th direction.

Measure and action are invariant under the gauge transformation

$$
\begin{aligned}
U(x ; \mu) & \rightarrow \Lambda(x) U(x ; \mu) \Lambda(x+a \widehat{\mu})^{-1} \\
\psi(x) & \rightarrow \Lambda(x) \psi(x) \\
\bar{\psi}(x) & \rightarrow \bar{\psi}(x) \Lambda(x)^{-1}
\end{aligned}
$$

with all $\Lambda(x) \in U(1)$. Furthermore, because of (7) they are invariant under the infinitesimal global chiral transformation

$$
\begin{aligned}
\delta \psi(x) & =i \epsilon \sigma_{\alpha} \gamma_{5}\left(1-\frac{a}{2} D\right) \psi(x) \\
\delta \bar{\psi}(x) & =i \epsilon \bar{\psi}\left(1-\frac{a}{2} D\right)(x) \gamma_{5} \sigma_{\alpha} .
\end{aligned}
$$

Finally, we observe that the theory is invariant under the charge conjugation

$$
\begin{aligned}
A_{\mu}(x) & \rightarrow-A_{\mu}(x), \\
\psi(x) & \rightarrow C \bar{\psi}(x)^{T} \\
\bar{\psi}(x) & \rightarrow \psi(x)^{T} \gamma_{0}^{T} C^{-1} \gamma_{0},
\end{aligned}
$$

where the superscript $T$ denotes transposition and $C$ the charge conjugation matrix satisfying

$$
C^{-1} \gamma_{\mu} C=-\gamma_{\mu}^{T}, \quad \mu=0, \ldots, 3
$$


For the perturbative evaluation we restrict our attention to a small neighbourhood of the pure gauge orbit. Here we parametrize

$$
U(x ; \mu)=\Lambda(x) \exp \left(i a g A_{\mu}(x)\right) \Lambda(x+a \widehat{\mu})^{-1},
$$

with the $A_{\mu}(x)$ subject to the Lorentz gauge

$$
F(A(x))=\sum_{\mu=0}^{3} \frac{1}{a} \widehat{\partial}_{\mu}^{*} A_{\mu}(x) \equiv 0 .
$$

Here and in the following, $\widehat{\partial}$ and $\widehat{\partial}^{*}$ denote the forward and backward lattice difference operators, respectively,

$$
\widehat{\partial}_{\mu} f(x)=f(x+a \widehat{\mu})-f(x), \widehat{\partial}_{\mu}^{*} f(x)=f(x)-f(x-a \widehat{\mu}) .
$$

Going through the standard gauge fixing and Faddeev-Popov procedure we end up with the following generating functional $W_{0}$ of the connected correlation function.

$$
\begin{gathered}
\exp \frac{1}{\hbar} W_{0}(J, \eta, \bar{\eta})=\mathcal{N}_{0} \int \prod_{x}\left(d \psi(x) d \bar{\psi}(x) \prod_{\mu} d A_{\mu}(x)\right) \\
\cdot \exp \left\{\mathcal{E}_{0}(A, \psi, \bar{\psi} ; g, \lambda)+\frac{1}{\hbar} S_{c}(A, \psi, \bar{\psi} ; J, \eta, \bar{\eta})\right\},
\end{gathered}
$$

with $W(0,0,0)=0$ and

$$
\mathcal{E}_{0}=-\frac{1}{\hbar}\left(S_{f}\left(e^{i a g A}, \psi, \bar{\psi}\right)+S_{W}\left(e^{i a g A}\right)+S_{g f}(A)\right) .
$$

$S_{g f}$ is the gauge fixing action

$$
S_{g f}(A)=a^{4} \sum_{x} \frac{\lambda}{2} F(A(x))^{2} .
$$

The source term $S_{c}$ is given by

$$
S_{c}(A, \psi, \bar{\psi} ; J, \eta, \bar{\eta})=a^{4} \sum_{x}\left\{\sum_{\mu} J_{\mu}(x) A_{\mu}(x)+\bar{\eta}(x) \psi(x)+\bar{\psi}(x) \eta(x)\right\} .
$$

Because we have chosen a linear gauge fixing function (17), the ghost fields decouple and do not need to be considered for the following considerations. In (19) we have introduced $\hbar$ mainly as the loop counting parameter. Any connected Feynman diagram with $n$ loops contributes to the order of $\hbar^{n}$ to $W$ or $\Gamma$. 


\section{$3 \quad$ Ward identities}

Applying an infinitesimal gauge transformation to (19),

$$
\begin{aligned}
\delta_{\omega} A_{\mu}(x) & =-\frac{1}{a} \widehat{\partial}_{\mu} \omega(x) \\
\delta_{\omega} \psi(x) & =i \omega(x) g \psi(x) \\
\delta_{\omega} \bar{\psi}(x) & =-i \omega(x) g \bar{\psi}(x),
\end{aligned}
$$

with $\omega(x) \in \mathbb{R}$, we obtain the Ward identity

$$
-i a^{4} \sum_{\mu} \frac{1}{a} \widehat{\partial}_{\mu}^{*} J_{\mu}(x)+\left[g \bar{\eta}(x) \frac{\partial}{\partial \bar{\eta}(x)}-g \eta(x) \frac{\partial}{\partial \eta(x)}-i \sum_{\mu} \lambda \frac{1}{a^{3}} \widehat{\square} \widehat{\partial}_{\mu}^{*} \frac{\partial}{\partial J_{\mu}(x)}\right] W_{0}=0,
$$

with $\widehat{\square}=\sum_{\mu} \widehat{\partial}_{\mu}^{*} \widehat{\partial}_{\mu}$. We perform a Legendre transformation to the vertex functional $\Gamma_{0}$, i.e. the generating functional of the one-particle irreducible (1PI) correlation functions,

$$
W_{0}(J, \eta, \bar{\eta})=\Gamma_{0}(\mathcal{A}, \psi, \bar{\psi})+a^{4} \sum_{x}\left(\sum_{\mu} J_{\mu}(x) \mathcal{A}_{\mu}(x)+\bar{\eta}(x) \psi(x)+\bar{\psi}(x) \eta(x)\right)
$$

where

$$
a^{4} \mathcal{A}_{\mu}(x)=\frac{\partial W_{0}}{\partial J_{\mu}(x)}, \quad a^{4} \psi(x)=\frac{\partial W_{0}}{\partial \bar{\eta}(x)}, \quad a^{4} \bar{\psi}(x)=-\frac{\partial W_{0}}{\partial \eta(x)},
$$

with inversion

$$
a^{4} J_{\mu}(x)=-\frac{\partial \Gamma_{0}}{\partial \mathcal{A}_{\mu}(x)}, \quad a^{4} \bar{\eta}(x)=\frac{\partial \Gamma_{0}}{\partial \psi(x)}, \quad a^{4} \eta(x)=-\frac{\partial \Gamma_{0}}{\partial \bar{\psi}(x)} .
$$

This yields the gauge Ward identity

$$
\mathcal{S}\left(\Gamma_{0}\right)(x)=0
$$

where

$$
\begin{aligned}
\mathcal{S}\left(\Gamma_{0}\right)(x) \equiv & i \sum_{\mu} \frac{1}{a} \widehat{\partial}_{\mu}^{*} \frac{\partial \Gamma_{0}}{\partial \mathcal{A}_{\mu}(x)}+\left[g \bar{\psi}(x) \frac{\partial \Gamma_{0}}{\partial \bar{\psi}(x)}-g \psi(x) \frac{\partial \Gamma_{0}}{\partial \psi(x)}\right] \\
& -i \lambda a \sum_{\mu} \widehat{\square} \widehat{\partial}_{\mu}^{*} \mathcal{A}_{\mu}(x)
\end{aligned}
$$

Furthermore, applying the chiral transformation (13) to (19) yields

$$
0=\prod_{x}\left(d \psi(x) d \bar{\psi}(x) \prod_{\mu} d A_{\mu}(x)\right) \exp \left(\frac{1}{\hbar} S_{c}\right)
$$




$$
\begin{gathered}
\cdot a^{4} \sum_{x}\left\{\bar{\eta}(x) \sigma_{\alpha} \gamma_{5}\left(\psi(x)+\hbar \frac{a}{2} \frac{\partial}{\partial a^{4} \bar{\psi}(x)}\right)\right. \\
\left.+\left(\bar{\psi}(x)-\hbar \frac{a}{2} \frac{\partial}{\partial a^{4} \psi(x)}\right) \gamma_{5} \sigma_{\alpha} \eta(x)\right\} \exp \mathcal{E}_{0} \\
=\prod_{x}\left(d \psi(x) d \bar{\psi}(x) \prod_{\mu} d A_{\mu}(x)\right) \exp \mathcal{E}_{0} \\
\cdot a^{4} \sum_{x}\left\{\bar{\eta}(x) \sigma_{\alpha} \gamma_{5}\left(\psi(x)-\hbar \frac{a}{2} \frac{\partial}{\partial a^{4} \bar{\psi}(x)}\right)\right. \\
\left.+\left(\bar{\psi}(x)+\hbar \frac{a}{2} \frac{\partial}{\partial a^{4} \psi(x)}\right) \gamma_{5} \sigma_{\alpha} \eta(x)\right\} \exp \left(\frac{1}{\hbar} S_{c}\right) .
\end{gathered}
$$

In the last step we have performed a partial integration. This expression can be written in the form

$$
a^{4} \sum_{x}\left\{\bar{\eta}(x) \sigma_{\alpha} \gamma_{5} \frac{\partial W_{0}}{\partial a^{4} \bar{\eta}(x)}-\frac{\partial W_{0}}{\partial a^{4} \eta(x)} \gamma_{5} \sigma_{\alpha} \eta(x)-\bar{\eta}(x) a \sigma_{\alpha} \gamma_{5} \eta(x)\right\}=0 .
$$

Application of the Legendre tranform (25) yields the flavour mixing, axial vector Ward identity

$$
\mathcal{C}\left(\Gamma_{0}\right)=-a^{4} \sum_{x} \frac{\partial \Gamma_{0}}{\partial a^{4} \psi(x)} a \sigma_{\alpha} \gamma_{5} \frac{\partial \Gamma_{0}}{\partial a^{4} \bar{\psi}(x)},
$$

where we have defined

$$
\mathcal{C}\left(\Gamma_{0}\right) \equiv a^{4} \sum_{x}\left\{\frac{\partial \Gamma_{0}}{\partial a^{4} \psi(x)} \sigma_{\alpha} \gamma_{5} \psi(x)-\bar{\psi}(x) \sigma_{\alpha} \gamma_{5} \frac{\partial \Gamma_{0}}{\partial a^{4} \bar{\psi}(x)}\right\} .
$$

The Ward identities (28) and (32) express the gauge invariance and the chiral symmetry of the bare lattice theory. The axial Ward identity contains a term that is bilinear in the vertex functional and classically irrelevant, i.e. with a coefficient that vanishes proportional to the lattice spacing $a$.

\section{Renormalization}

We write the renormalized generating functional of connected correlation functions $W_{R}$ as

$$
\begin{gathered}
\exp \frac{1}{\hbar} W_{R}(J, \eta, \bar{\eta})=\mathcal{N}_{R} \int \prod_{x}\left(d \psi(x) d \bar{\psi}(x) \prod_{\mu} d A_{\mu}(x)\right) \\
\cdot \exp \left\{\mathcal{E}_{R}(A, \psi, \bar{\psi} ; g, \lambda)+\frac{1}{\hbar} S_{c}(A, \psi, \bar{\psi} ; J, \eta, \bar{\eta})\right\}
\end{gathered}
$$


with $W_{R}(0,0,0)=0$ and

$$
\mathcal{E}_{R}(A, \psi, \bar{\psi} ; g, \lambda)=\mathcal{E}_{0}(A, \psi, \bar{\psi} ; g, \lambda)+O\left(\hbar^{0}\right)
$$

The renormalized vertex functional $\Gamma_{R}$ is obtained from $W_{R}$ by the Legendre transformation analogous to (25). By definition, all basic field $(A, \psi, \bar{\psi})$ correlation functions exist in the continuum limit. In particular, this means that the connected correlation functions

$$
\begin{aligned}
& G_{r, s, l-r-s}\left(\left(z_{1}, \mu_{1}\right), \ldots ; z_{r+1}, \ldots ; z_{r+s+1}, \ldots, z_{l} \mid a\right) \\
& \equiv a^{4 l} \sum_{x_{1}, \ldots, x_{l} \in a \mathbb{Z}^{4}} \prod_{i=1}^{l} \delta^{4}\left(z_{i}-x_{i}\right) \cdot<A_{\mu_{1}}\left(x_{1}\right) \cdots \psi\left(x_{r+1}\right) \cdots \bar{\psi}\left(x_{l}\right)>_{c}
\end{aligned}
$$

with

$$
\begin{aligned}
& <A_{\mu_{1}}\left(x_{1}\right) \cdots \psi\left(x_{r+1}\right) \cdots \bar{\psi}\left(x_{l}\right)>_{c} \\
& =\left.\hbar^{l} \frac{\partial}{\partial a^{4} J_{\mu_{1}}\left(x_{1}\right)} \cdots \frac{\partial}{\partial a^{4} \bar{\eta}\left(x_{r+1}\right)} \cdots \frac{\partial}{\partial a^{4}\left(-\eta\left(x_{l}\right)\right)} \frac{1}{\hbar} W_{R}\right|_{J=\eta=\bar{\eta}=0}
\end{aligned}
$$

converge for $a \rightarrow 0$ as tempered distributions on $\mathbb{R}^{4 l}$.

The renormalizability statement below is that $\mathcal{E}_{R}$ is obtained from $\mathcal{E}_{0}$ by multiplicative renormalization of its arguments and such that both gauge invariance and lattice chiral symmetry are preserved.

Theorem. There exist renormalization constants $Z_{A}, Z_{\psi}, Z_{g}$ and $Z_{\lambda}$ such that

$$
\mathcal{E}_{R}(A, \psi, \bar{\psi} ; g, \lambda)=\mathcal{E}_{0}\left(Z_{A}^{1 / 2} A, Z_{\psi}^{1 / 2} \psi, Z_{\psi}^{1 / 2} \bar{\psi} ; Z_{g} g, Z_{\lambda} \lambda\right)
$$

with

$$
Z_{A} Z_{g}^{2}=Z_{A} Z_{\lambda}=1
$$

The renormalization constants are at most logarithmically divergent as $a \rightarrow 0$.

As a consequence of the theorem, the renormalized vertex functional $\Gamma_{R}$ satisfies the gauge and axial Ward identities

$$
\begin{aligned}
\mathcal{S}\left(\Gamma_{R}\right) & =0 \\
\mathcal{C}\left(\Gamma_{R}\right) & =a^{4} \sum_{x} \xi(a) \frac{\partial \Gamma_{R}}{\partial a^{4} \psi(x)} a \sigma_{\alpha} \gamma_{5} \frac{\partial \Gamma_{R}}{\partial a^{4} \bar{\psi}(x)},
\end{aligned}
$$

where $\xi(a)$ is at most logarithmically divergent as $a \rightarrow 0$.

The two independent renormalization constants $Z_{A}$ and $Z_{\psi}$ are uniquely determined by imposing two independent normalization conditions, e.g. on the 2-point functions, at non-exceptional momenta.

The statement implies that abelian lattice gauge theory with massless Ginsparg Wilson fermions is renormalizable, preserving gauge and chiral symmetry. In particular, no mass counterterm for the fermion fields is required. The irrelevant part 
of the lattice axial Ward identity gets multiplicatively renormalized, but in such a way that it stays irrelevant to all orders. This means that the continuum chiral symmetry becomes restored in the continuum limit (that is, (2) holds). As a corollary, the current $j_{\mu}(x)$ associated to the symmetry (13) and constructed according to the Poincare lemma on the lattice [9] does not require renormalization (cf. also [10]). For, if we write the variation of the action under a local chiral transformation

$$
\delta \psi(x)=i \epsilon(x) \sigma_{\alpha} \gamma_{5}\left[\left(1-\frac{a}{2} D\right) \psi\right](x), \quad \delta \bar{\psi}(x)=i \epsilon(x)\left[\bar{\psi}\left(1-\frac{a}{2} D\right)\right](x) \gamma_{5} \sigma_{\alpha}
$$

as

$$
\delta \mathcal{E}_{R}=\frac{1}{\hbar} a^{4} \sum_{x} i \epsilon(x)\left(\sum_{\mu} \frac{1}{a} \widehat{\partial}_{\mu}^{*} j_{\mu \alpha}(x)\right)
$$

and add to the source part of the action $S_{c}$ a term

$$
a^{4} \sum_{x, \mu} \sum_{\alpha=1}^{3} G_{\mu \alpha}(x) j_{\mu \alpha}(x),
$$

the corresponding vertex functional $\Gamma_{R}^{\prime}(A, \psi, \bar{\psi} ; G)$ satisfies the axial vector current Ward identity

$$
\begin{gathered}
\sum_{\mu} \frac{1}{a} \widehat{\partial}_{\mu}^{*} \frac{\partial \Gamma_{R}^{\prime}}{\partial a^{4} G_{\mu \alpha}(x)}=-\left\{\frac{\partial \Gamma_{R}^{\prime}}{\partial a^{4} \psi(x)} \sigma_{\alpha} \gamma_{5} \psi(x)-\bar{\psi}(x) \sigma_{\alpha} \gamma_{5} \frac{\partial \Gamma_{R}^{\prime}}{\partial a^{4} \bar{\psi}(x)}\right\} \\
+\xi(a) \frac{\partial \Gamma_{R}^{\prime}}{\partial a^{4} \psi(x)} a \sigma_{\alpha} \gamma_{5} \frac{\partial \Gamma_{R}^{\prime}}{\partial a^{4} \bar{\psi}(x)}+O(G)
\end{gathered}
$$

Because of $\Gamma_{R}^{\prime}(A, \psi, \bar{\psi} ; G=0)=\Gamma_{R}(A, \psi, \bar{\psi})$, the right hand side of (44) is UV finite to order $G^{0}$, and hence also the left hand side. This implies that all correlation functions with one insertion of the composite operator $j_{\mu}(x)$ are made UV finite already by renormalizing the gauge theory.

\section{Proof of the theorem}

For the proof we use the lattice power counting theorem. Its applicability to gauge theories with Ginsparg Wilson fermions is guaranteed by

- The action has the correct continuum limit.

- Analyticity of the Fourier transforms of the coefficient functions $D^{(n)}$ in (8) at zero momenta.

- The free Ginsparg Wilson propagator $\widetilde{D}_{0}(k)^{-1}$ is free of doublers. 
More precisely, the last point means that $\widetilde{D}_{0}(k)^{-1}$ is of the form

$$
\widetilde{D}_{0}(k)^{-1}=\frac{\mathcal{N}(k)}{\mathcal{R}(k)},
$$

where $\mathcal{N}$ and $\mathcal{R}$ satisfy the following conditions.

$$
\begin{aligned}
& \lim _{a \rightarrow 0} \mathcal{N}(k)=-i \sum_{\mu=0}^{3} \gamma_{\mu} k_{\mu}, \quad \overline{\operatorname{degr}}_{\widehat{k}} \mathcal{N}(k)=1, \\
& \lim _{a \rightarrow 0} \mathcal{R}(k)=k^{2}, \quad \overline{\operatorname{degr}}_{\widehat{k}} \mathcal{R}(k)=2
\end{aligned}
$$

and for sufficiently small $a$ there is $K>0$ such that

$$
\mathcal{R}(k) \geq K \widehat{k}^{2}
$$

$\overline{\operatorname{degr}}_{\widehat{k}}(\cdots)$ denotes the (UV) lattice divergence degree [8] and

$$
\widehat{k}^{2}=\sum_{\mu=0}^{3} \widehat{k}_{\mu}^{2}, \quad \widehat{k}_{\mu}=\frac{2}{a} \sin \frac{k_{\mu}}{2} a .
$$

It is straightforward to show that the propagator given in [5], with

$$
\begin{aligned}
& \mathcal{N}(k)=\mathcal{J}(k)^{1 / 2}\left[\frac{1}{a}\left(\mathcal{J}(k)^{1 / 2}-1+\frac{a^{2}}{2} \widehat{k}^{2}\right)-i \sum_{\mu} \gamma_{\mu} \widetilde{k}_{\mu}\right], \\
& \mathcal{R}(k)=\widetilde{k}^{2}+\frac{1}{a^{2}}\left(\mathcal{J}(k)^{1 / 2}-1+\frac{a^{2}}{2} \widehat{k}^{2}\right)^{2}, \\
& \mathcal{J}(k)=1+\frac{a^{4}}{4} \sum_{\mu \neq \nu} \widehat{k}_{\mu}^{2} \widehat{k}_{\nu}^{2}, \\
& \widetilde{k}^{2}=\sum_{\mu=0}^{3} \widetilde{k}_{\mu}^{2}, \quad \widetilde{k}_{\mu}=\frac{1}{a} \sin k_{\mu} a,
\end{aligned}
$$

satisfies the criteria (46) and (47) with $K=1$.

The proof of the theorem is by induction on the number of loops, that is on the order of $\hbar$. To lowest order, the theorem holds, with all renormalization constants $Z_{A}=Z_{\psi}=Z_{g}=Z_{\lambda}=1$, i.e. all tree graph amplitudes are UV finite. Furthermore $\xi(a)=-1$.

Let us assume that the renormalization program has been carried out to order $n$ in $\hbar$, with renormalized action given by

$$
\mathcal{E}_{R}^{(n)}(A, \psi, \bar{\psi} ; g, \lambda)=\mathcal{E}_{0}\left(Z_{A}^{(n)^{1 / 2}} A, Z_{\psi}^{(n)^{1 / 2}} \psi, Z_{\psi}^{(n)^{1 / 2}} \bar{\psi} ; Z_{g}^{(n)} g, Z_{\lambda}^{(n)} \lambda\right)
$$

and

$$
Z_{A}^{(n)} Z_{g}^{(n)^{2}}=Z_{A}^{(n)} Z_{\lambda}^{(n)}=1
$$


with $Z_{A}^{(n)}$ and $Z_{\psi}^{(n)}$ at most logarithmically UV divergent. Hence, the vertex functional $\Gamma_{R}^{(n)}$ renormalized to order $n$ satisfies

$$
\begin{aligned}
\mathcal{S}\left(\Gamma_{R}^{(n)}\right) & =0 \\
\mathcal{C}\left(\Gamma_{R}^{(n)}\right) & =a^{4} \sum_{x} \xi^{(n)}(a) \frac{\partial \Gamma_{R}^{(n)}}{\partial a^{4} \psi(x)} a \sigma_{\alpha} \gamma_{5} \frac{\partial \Gamma_{R}^{(n)}}{\partial a^{4} \bar{\psi}(x)},
\end{aligned}
$$

with $\xi^{(n)}(a)=-Z_{\psi}^{(n)^{-1}}$. Considered as perturbative series in $\hbar, \xi^{(n)}(a)$ is at most logarithmically divergent.

Power counting and the properties of the lattice action listed above ensure that all vertices derived from it have IR degree not less than 4 and UV degree equal to 4. This implies that IR and UV singularities are well separated and that the theory is renormalizable in an IR finite way [11]. The most general UV divergence of $\Gamma_{R}^{(n)}$ of order $\hbar^{n+1}$, which we denote by $\Gamma_{\text {div }}$ below, is a polynomial in the fields and their lattice derivatives, obtained by accounting for all 1PI correlation functions with non-negative UV divergence degree. Taking into account the lattice symmetries, it is given by

$$
\begin{aligned}
\Gamma_{d i v}= & a^{4} \sum_{x}\left\{\delta m_{\psi} \bar{\psi}(x) \psi(x)+r_{\psi} \sum_{\mu} \bar{\psi}(x) \gamma_{\mu} \frac{1}{a} \widehat{\partial}_{\mu} \psi(x)\right. \\
+ & r_{\bar{\psi}} A \psi \sum_{\mu} g \bar{\psi}(x) \gamma_{\mu} A_{\mu}(x) \psi(x)+\sum_{\mu} \frac{1}{2} \delta m_{A}^{2} A_{\mu}(x)^{2} \\
+ & \sum_{\mu \nu}\left[\frac{1}{4} r_{A}\left(\frac{1}{a} \widehat{\partial}_{\mu} A_{\nu}(x)-\frac{1}{a} \widehat{\partial}_{\nu} A_{\mu}(x)\right)^{2}\right. \\
+ & \frac{1}{2}\left(r_{g f}+\delta_{\mu \nu} r_{2}\right)\left(\frac{1}{a} \widehat{\partial}_{\mu} A_{\mu}(x)\right)\left(\frac{1}{a} \widehat{\partial}_{\nu} A_{\nu}(x)\right) \\
+ & \left.\left.\frac{1}{4}\left(r_{41}+\delta_{\mu \nu} r_{42}\right) g^{2} A_{\mu}(x)^{2} A_{\nu}(x)^{2}\right]\right\} .
\end{aligned}
$$

All coefficients are $O\left(\hbar^{n+1}\right)$ and all those denoted by $r_{\bullet}$ are at most logarithmically divergent as $a \rightarrow 0$. Inserting this into the gauge Ward identity

$$
\mathcal{S}\left(\Gamma_{d i v}\right)(x)=O(1) \quad \text { as } a \rightarrow 0
$$

we obtain the constraints

$$
\begin{aligned}
& r_{g f}=r_{2}=r_{41}=r_{42}=\delta m_{A}^{2}=0, \\
& r_{\bar{\psi} A \psi}=i r_{\psi} .
\end{aligned}
$$

From the axial Ward identity

$$
\mathcal{C}\left(\Gamma_{\text {div }}\right)=O(1) \quad \text { as } a \rightarrow 0
$$


we get the further constraint $\delta m_{\psi}=0$. In summary, the UV divergencies of order $\hbar^{n+1}$ are removed by adding to $\hbar \mathcal{E}_{0}$ the counterterm

$$
\begin{aligned}
\mathcal{E}_{C T} & =-a^{4} \sum_{x}\left\{r_{\psi} \sum_{\mu} \bar{\psi}(x) \gamma_{\mu}\left(\frac{1}{a} \widehat{\partial}_{\mu}+i g A_{\mu}(x)\right) \psi(x)\right. \\
& \left.+\sum_{\mu \nu} \frac{1}{4} r_{A}\left(\frac{1}{a} \widehat{\partial}_{\mu} A_{\nu}(x)-\frac{1}{a} \widehat{\partial}_{\nu} A_{\mu}(x)\right)^{2}\right\}
\end{aligned}
$$

With $r_{\lambda}=-r_{A}$ and $r_{g}=-r_{A} / 2$ we write this as

$$
\begin{aligned}
& \mathcal{E}_{C T}=\left(\frac{r_{A}}{2} \frac{\partial}{\partial \tau_{A}}+r_{g} \frac{\partial}{\partial \tau_{g}}+\frac{r_{\psi}}{2}\left(\frac{\partial}{\partial \tau_{\psi}}+\frac{\partial}{\partial \tau_{\bar{\psi}}}\right)+r_{\lambda} \frac{\partial}{\partial \tau_{\lambda}}\right) \\
& \cdot\left(-a^{4}\right) \sum_{x}\left\{\frac{1}{4} \sum_{\mu \nu} \tau_{A}^{2}\left(\frac{1}{a} \widehat{\partial}_{\mu} A_{\nu}(x)-\frac{1}{a} \widehat{\partial}_{\nu} A_{\mu}(x)\right)^{2}\right. \\
&+\tau_{\psi} \tau_{\bar{\psi}} \bar{\psi}(x) \sum_{\mu} \gamma_{\mu}\left(\frac{1}{a} \widehat{\partial}_{\mu}+i \tau_{A} \tau_{g} g A_{\mu}(x)\right) \psi(x) \\
&\left.+\frac{\lambda}{2} \tau_{A}^{2} \tau_{\lambda}\left(\sum_{\mu} \frac{1}{a} \widehat{\partial}_{\mu}^{*} A_{\mu}(x)\right)^{2}\right\}_{\tau \equiv 1},
\end{aligned}
$$

so that

$$
\begin{aligned}
\mathcal{E}_{R}^{(n)}+\frac{1}{\hbar} \mathcal{E}_{C T} & =\mathcal{E}_{0}\left(Z_{A}^{(n+1)^{1 / 2}} A, Z_{\psi}^{(n+1)^{1 / 2}} \psi, Z_{\psi}^{(n+1)^{1 / 2}} \bar{\psi} ; Z_{g}^{(n+1)} g, Z_{\lambda}^{(n+1)} \lambda\right) \\
& +\frac{1}{\hbar} \Delta
\end{aligned}
$$

with $Z_{i}^{(n+1)}=Z_{i}^{(n)}+r_{i}$ for $i=A, \psi$ and with $Z_{g}^{(n+1)}=Z_{A}^{(n+1)^{-1 / 2}}, Z_{\lambda}^{(n+1)}=$ $Z_{A}^{(n+1)^{-1}} . \Delta$ is a local lattice operator of IR degree not less than 4 , UV degree 4 , and it is UV finite and irrelevant up to and including order $\hbar^{n+1}$. We are thus allowed to subtract it from $\mathcal{E}_{R}^{(n)}+\frac{1}{\hbar} \mathcal{E}_{C T}$. This yields the action renormalized to the order $\hbar^{n+1}$, satisfying

$$
\begin{aligned}
& \mathcal{E}_{R}^{(n+1)}(A, \psi, \bar{\psi} ; g, \lambda) \\
& \quad=\mathcal{E}_{0}\left(Z_{A}^{(n+1)^{1 / 2}} A, Z_{\psi}^{(n+1)^{1 / 2}} \psi, Z_{\psi}^{(n+1)^{1 / 2}} \bar{\psi} ; Z_{g}^{(n+1)} g, Z_{\lambda}^{(n+1)} \lambda\right)
\end{aligned}
$$

where the $Z_{i}^{(n+1)}$ fulfill (51) with $n$ replaced by $n+1$. This closes the induction and proves the theorem.

The above proof was carried out for an abelian gauge theory. For QCD with two flavours of massless Ginsparg-Wilson fermions the axial vector Ward identity is again of the form (32). The main complications arise due to the compact gauge field 
measure on the lattice and from the non-linear structure of the gauge Ward identity. The renormalizability proof proceeds along similar lines as in [0], supplemented by the axial vector Ward identity, whose renormalized version (40) is obtained by following the procedure described above.

\section{Acknowledgment}

One of us (T.R.) would like to thank Jean Zinn-Justin for encouraging discussions.

\section{References}

[1] W. Bietenholz, U.-J. Wiese, Phys. Lett. B378 (1996) 222;

Nucl. Phys. (Proc. Suppl.) 47 (1996) 575.

[2] H. Neuberger, Phys. Lett. B417 (1998) 141; ibid B427 (1998) 353.

[3] H. B. Nielsen and M. Ninomiya, Nucl. Phys. B185 (1981) 20; ibid B193 (1981) 173.

[4] P. Ginsparg and K. Wilson, Phys. Rev. D25 (1982) 2649.

[5] M. Lüscher, Phys. Lett. B428 (1998) 342.

[6] P. Hernández, K. Jansen, M. Lüscher, Nucl. Phys. B552 (1999) 363

[7] T. Reisz, Nucl. Phys. B318 (1989) 417.

[8] T. Reisz, Commun. Math. Phys. 116 (1988) 81; ibid 117 (1988) 79; M. Lüscher, "Selected Topics in Lattice Field Theory", Lectures given at the Summer school on Fields, Strings and Critical Phenomena, Les Houches (1988); H. J. Rothe, "Lattice Gauge Theories: An Introduction", Second Edition, World Scientific Lecture Notes in Physics Vol. 59, World Scientific 1997.

[9] M. Lüscher, Nucl. Phys. B538 (1999) 515.

[10] P. Hasenfratz, Nucl. Phys. B525 (1998) 401.

[11] J. H. Lowenstein, W. Zimmermann, Nucl. Phys. B86 (1975) 77; J. H. Lowenstein, Commun. Math. Phys. 47 (1976) 53; T. Reisz, Commun. Math. Phys. 117 (1988) 639. 